\title{
Convergent validity and reliability of a novel repeated agility protocol in junior rugby league players [version 1; peer
}

\section{review: 2 approved with reservations]}

\author{
Anthony Nicholls (D1, Anthony Leicht (D1) , Jonathan Connor (D1), Aaron Halliday2, \\ Kenji Doma (iD)1
}

${ }^{1}$ Sport \& Exercise Science, James Cook University, Douglas, Queensland, 4814, Australia

2Physical Education, Kirwan State Highschool, Kirwan, Queensland, 4817, Australia

V1 First published: 17 Jun 2020, 9:624

https://doi.org/10.12688/f1000research.23129.1

Second version: 08 Oct 2021, 9:624

https://doi.org/10.12688/f1000research.23129.2

Latest published: 22 Nov 2021, 9:624

https://doi.org/10.12688/f1000research.23129.3

\section{Abstract}

Background: Rugby league involves repeated, complex, change-ofdirection movements, although there are no test protocols that specifically assesses these physical fitness profiles. Thus, the current study examined the convergent validity and reliability of a repeated Illinois Agility (RIA) protocol in adolescent Rugby League players. Methods: Twenty-two junior Rugby League players completed 4 sessions with each separated by 7 days. Initially, physical fitness characteristics at baseline (i.e., multi-stage fitness, countermovement jump, 30-m sprint, single-effort agility and repeated sprint ability [RSA]) were assessed. The second session involved a familiarisation of RIA and repeated T-agility test (RTT) protocols. During the third and fourth sessions, participants completed the RIA and RTT protocols in a randomised, counterbalanced design to examine the validity and testretest reliability of these protocols.

Results: For convergent validity, significant correlations were identified between RIA and RTT performances $(r=>0.80 ; p<0.05)$. For contributors to RIA performance, significant correlations were identified between all baseline fitness characteristics and RIA ( $r=$ $>0.71 ; p<0.05$ ). Reliability of the RIA protocol was near perfect with excellent intra-class correlation coefficient (0.87-0.97), good ratio limits of agreement $(x / \div 1.05-1.06)$ and low coefficient of variations (1.77-1.97\%).

Conclusions: The current study has demonstrated the RIA to be a simple, valid and reliable field test that can provide coaches with information about their athlete's ability to sustain high intensity, multi-directional running efforts.

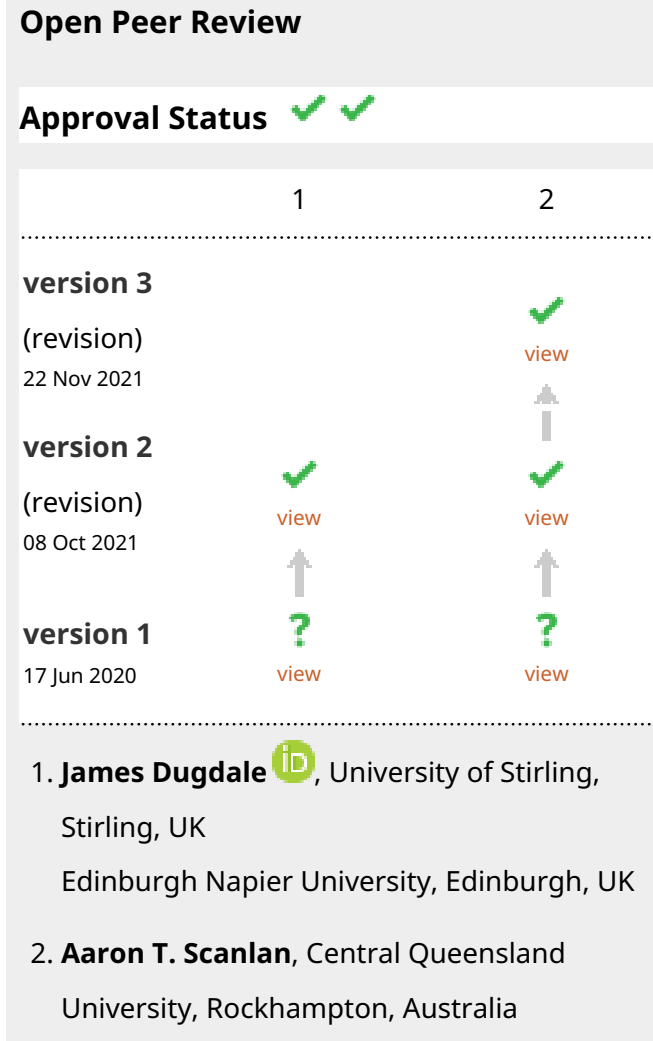

Any reports and responses or comments on the article can be found at the end of the article. 
Keywords

Change of direction, anaerobic power, sprint, speed, recovery rate

\section{Corresponding author: Kenji Doma (kenji.doma@jcu.edu.au)}

Author roles: Nicholls A: Data Curation, Formal Analysis, Investigation, Methodology, Project Administration, Writing - Original Draft Preparation, Writing - Review \& Editing; Leicht A: Conceptualization, Formal Analysis, Methodology, Project Administration, Supervision, Writing - Original Draft Preparation, Writing - Review \& Editing; Connor J: Methodology, Project Administration, Supervision, Writing Review \& Editing; Halliday A: Methodology, Project Administration, Supervision, Writing - Review \& Editing; Doma K: Conceptualization, Data Curation, Formal Analysis, Investigation, Methodology, Project Administration, Software, Supervision, Writing - Original Draft Preparation, Writing - Review \& Editing

Competing interests: No competing interests were disclosed.

Grant information: The author(s) declared that no grants were involved in supporting this work.

Copyright: ( 2020 Nicholls A et al. This is an open access article distributed under the terms of the Creative Commons Attribution License, which permits unrestricted use, distribution, and reproduction in any medium, provided the original work is properly cited.

How to cite this article: Nicholls A, Leicht A, Connor J et al. Convergent validity and reliability of a novel repeated agility protocol in junior rugby league players [version 1; peer review: 2 approved with reservations] F1000Research 2020, 9:624 https://doi.org/10.12688/f1000research.23129.1

First published: 17 Jun 2020, 9:624 https://doi.org/10.12688/f1000research.23129.1 


\section{Introduction}

Rugby League (RL) is an intermittent, invasion type game that requires players to complete repetitive bursts of sprinting and multi-directional movements in response to the dynamic constraints of the game', typically referred to as 'agility' movements ${ }^{2}$. Traditionally, the physical component of agility has been assessed using change-of-direction routes with shorter time of completion considered a strong determinant of agility performance ${ }^{3}$. Some commonly used agility tests have included the Agility T-Test and the Illinois Agility test with both employed in many intermittent sports ${ }^{4}$. However, these two assessment protocols employ a single bout approach for the agility performance measure ${ }^{4,5}$. Athletes in RL encounter repeated bursts of change-of-direction movements to defend or evade defenders during a game $^{6}$. Consequently, performance of repeated agility activities with brief periods of rest may be an important performance component necessary for RL athletes.

As a monitoring tool, the reliability of repeated agility protocols have been explored in a variety of sports $^{7-9}$. Results from a study examining a Repeated T-Test (RTT) agility protocol in soccer players significantly correlated with anaerobic measures of power, speed and repeat-sprint ability (RSA), with excellent test-retest reliability ${ }^{9}$. While a good indicator of agility, the Agility T-Test consists of a linear sprint, lateral shuffles and a backwards run, which are movements that are sporadic in $\mathrm{RL}^{9}$. In fact, RL players change direction frequently and utilise evading movements ${ }^{5}$ that are not replicated by the Agility T-Test. Therefore, the Illinois Agility test may be more reflective of the evading activities undertaken in RL, as the protocol includes vigorous changes in direction by weaving in and out of cones ${ }^{4}$. As the validity and reliability of the Repeated Illinois Agility (RIA) test have yet to be determined, reporting these properties would be essential for widespread usability ${ }^{3}$.

The aims of this study were three-fold: 1) to examine the convergent validity of a novel RIA test with the repeated Agility T-test protocol (i.e. RTT); 2) to identify contributors of RIA performance by comparing its measures to speed, anaerobic capacity and recovery dynamics (i.e. RSA); and 3) to determine the test-retest reliability of the RIA protocol. It was hypothesised that the RIA would demonstrate acceptable convergent validity and reliability as a repeated agility test, with relationships identified between results of the RIA and the RTT, speed, and anaerobic capacity protocols. Identification of the convergent validity and reliability of the RIA will provide coaches with a tool to assist in monitoring and training RL athletes as well as in talent development and identification.

\section{Methods}

\section{Research design}

The current study was a randomised, counter-balanced study conducted across five sessions from June, 2018 to August, 2018 (Figure 1). During the first session, the participants completed a Multistage Shuttle test to determine predicted maximal aerobic capacity $\left(\mathrm{VO}_{2 \max }\right)^{10}$. The second session was utilised to obtain baseline assessments of speed (30-metre sprint), agility (Illinois Agility test, Agility T-Test) and repeat-sprint ability (RSA). The third session familiarised participants with the RTT and RIA tests. During the fourth and fifth sessions, participants undertook both the RIA and RTT, in randomised order, with at least 15-minutes of recovery between each protocol.

At the start of each session, muscle soreness rating was collected prior to performing a standardised warm up, using a 1-10 visual analogue scale, with 1 and 10 indicating 'no soreness' and "very, very sore" ${ }^{11}$. Participants then performed a progressive warm-up consisting of jogging for 3-5 minutes and 15 -metre sprints at $50 \%, 70 \%$ and $100 \%$ of maximal effort. A countermovement jump (CMJ) test (Yard Stick, Swift Performance, Queensland, Australia) was then conducted to assess leg power ${ }^{12}$, which was also repeated before the second agility test to confirm recovery between the repeated agility tests.

\section{Participants}

In total, 22 adolescent, male, RL players (age $16.2 \pm 0.8 \mathrm{yrs}$; body mass $80.7 \pm 16.3 \mathrm{~kg}$; height $1.77 \pm 0.7 \mathrm{~m}$ ) who engaged in the School of Athletic Excellence program were recruited via word of mouth, flyers and liaison with sporting teams. The participants were injury-free with at least 2 years of RL experience. According to an a priori calculation ${ }^{13}$, a sample size of 22 was sufficient to identify significant differences in repeated-agility

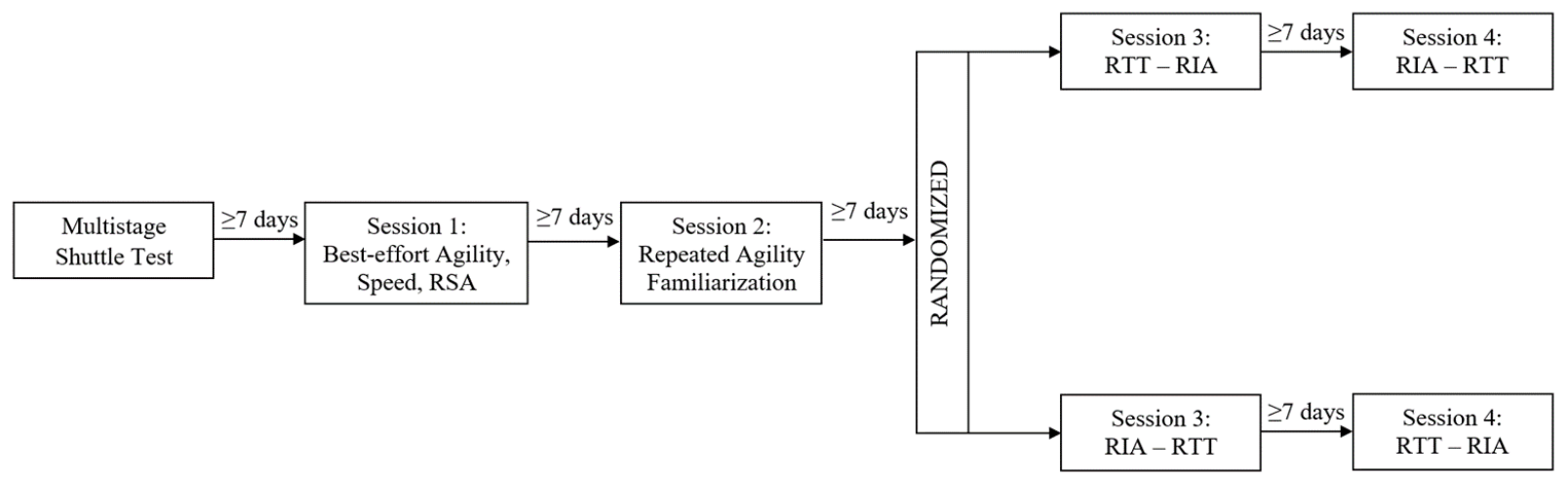

Figure 1. Schematic of the research design consisting of four sessions, including best-effort agility, speed, repeat sprint ability (RSA), repeated T-test agility (RTT) and repeated Illinois agility (RIA) measures. 
performance (power of $80 \%$, alpha level of 0.05 ). Participants were instructed to avoid strenuous physical activity and caffeine for up to 12 hours before each testing session. All protocols were approved by the Institutional Human Research Ethics Committee and written informed consent was received from the participants and their parents/guardians prior to partaking this study (Approval number H7248).

\section{Multistage shuttle test}

For the multistage shuttle test, participants ran back and forth in time with a series of audio signals on a $20 \mathrm{~m}$ indoor $\operatorname{court}^{10}$. The time between audio signals progressively decreased during the test resulting in an increased effort and running speed for athletes each minute. Predicted $\mathrm{VO}_{2 \max }$ was estimated using a previously developed regression equation ${ }^{10}$.

\section{Countermovement jump test}

The countermovement jump protocol was measured with a vertical jump apparatus, based on $1 \mathrm{~cm}$ increments (Yard Stick, Swift Performance, Queensland, Australia). To ensure standardisation of the countermovement jump test, participants were instructed to draw their arms backwards upon the eccentric phase, then swing the arms forward during the concentric phase to gain momentum and maximise the stretchshortening cycle mechanics ${ }^{14}$. The participants attempted three countermovement jumps, with approximately 30-60 seconds of rest in-between, and the highest jump reported.

\section{0-m Sprint and Agility protocols}

Assessment of speed was achieved by completing 30-m maximal sprints, the Agility T-test protocol was set up within a $10-\mathrm{m} \times$ 10 -m figure-T course (Figure 2A), and the Illinois Agility protocol consisted of a $10 \mathrm{~m} \times 5 \mathrm{~m}$ course (Figure $2 \mathrm{~B})^{3}$. To ensure protocol familiarity, the participants completed three trials at sub-maximal effort followed by one final maximal trial, with each trial interspersed by two minutes of recovery. Trial completion times were recorded using an electronic timing gate system (Speedlight Timing Gates, Swift Performance, Australia) positioned at the start/finishing line. The fastest time was used for later analysis.

\section{Repeat Sprint and Agility Protocols}

The RSA, RTT and RIA protocols were completed by repeating the previously described protocols (i.e. 30-m sprint, T-test and Illinois Agility, respectively) across 6 cycles with varying recovery periods in-between each cycle. Specifically, each cycle within the RSA, RTT and RIA was separated by 20-, 35- and 60-second recovery, respectively, with work-to-rest ratios of approximately $1: 3^{8,9}$. Immediately after each repeated agility cycle, participant's heart rate (HR, Polar
A)

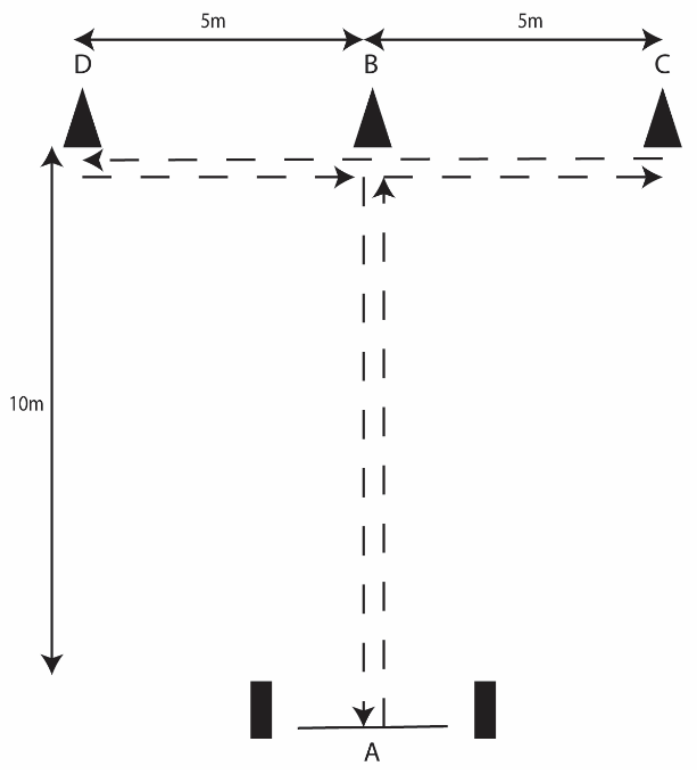

B)

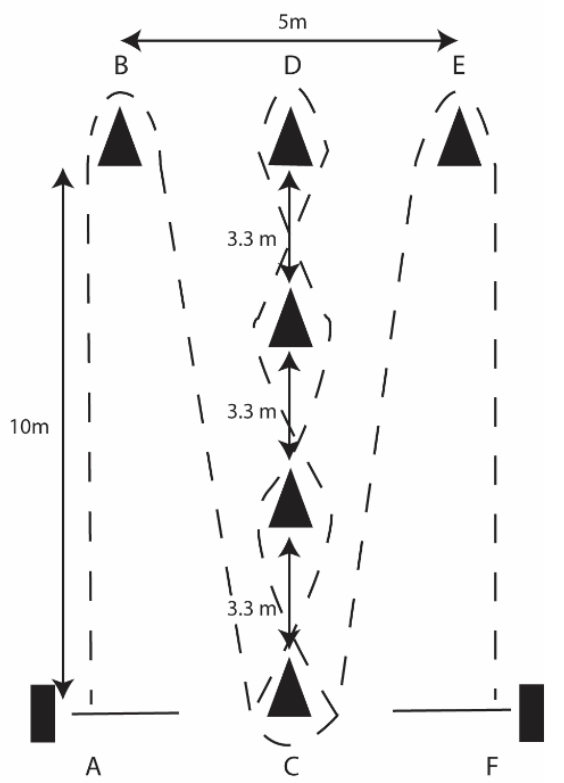

Timing Gates

Cones

Figure 2. Schematic of A) T-Test Agility and B) Illinois Agility protocols. 
Heart Rate Monitor, Polar H10, Finland) and maximum rating of perceived-exertion (RPE, Borg category scale 1-10) were then averaged across the 6 cycles for analysis ${ }^{15}$. The following parameters were also calculated for each repeated agility protocol: total time (TT) of 6 cycles, best cycle time (BT), the average cycle time (AT) and fatigue index $(\mathrm{FI})^{8}$. FI was calculated as follows :

$$
\text { Fatigue Index }=\left(\left(\frac{T T}{B T \times 6}\right) \times 100\right)-100
$$

\section{Statistical analysis}

Data was analysed using a statistical software (IBM SPSS version 25, Chicago, Illinois) and reported as mean \pm standard deviation. Normality of the data was assessed using the Kolmogorov-Smirnov statistic. Convergent validity of the repeated agility protocols was identified via Pearson's product moment correlation coefficients for RTT and RIA measures (i.e., TT, BT, AT and FI) and construct validity with aerobic capacity, leg power, speed and agility variables (i.e., $\mathrm{VO}_{2 \max }, \mathrm{CMJ}$, $30-\mathrm{m}$ sprint time, T-Test and Illinois Agility, respectively). The cut-off for acceptable convergent validity was established when the association was statistically significant with an r-value of $\geq 0.50^{16}$. Reliability of the repeated agility measures was determined via a paired T-test, intraclass correlation coefficients (ICC, SPSS 2-way mixed, 95\% confidence intervals), coefficient of variation (CV, 95\% confidence intervals) and systematic bias/ ratio with $95 \%$ limits of agreement $(\mathrm{LOA})^{17}$. Where significant relationships existed between the mean difference and average of test-retest values (i.e. heteroscedastic errors), variables were transformed (natural logarithm) prior to the calculation of measurement bias/ratio $\times / \div$ ratio LOA $^{18}$. The level of significance for all analyses was set at 0.05 . Finally, effect size (Cohen's $d$ ) with $95 \%$ CI was used to calculate the magnitude of differences in muscle soreness and CMJ measures between RIA and RTT protocols to determine whether the recovery periods were appropriate. The ES classifications were set as small, moderate and large with values of $0.2,0.5$ and 0.8 , respectively (Cohen, 1988).

\section{Results}

For convergent validity, significant correlations were identified between RIA and most RTT variables (Table $1^{19}$ ). For contributors to RIA performance, significant correlations were identified with RSA, 30-m sprint time, best effort agility measures, aerobic capacity and CMJ (Table $2^{19}$ ).

Muscle soreness ratings between the third $(2.0 \pm 1.5)$ and fourth $(2.6 \pm 1.7)$ were not significantly different $(\mathrm{p}=0.10)$, with a small ES (0.37). Jump height prior to each repeated agility protocol remained unchanged between the first and second CMJ tests in the third $(43.8 \mathrm{~cm} \pm 8.7 \mathrm{~cm}$ and $45.4 \mathrm{~cm} \pm$ $8.4 \mathrm{~cm}$, respectively, $\mathrm{p}=0.09)$ and fourth $(44.1 \mathrm{~cm} \pm 9.3 \mathrm{~cm}$ and $44.0 \mathrm{~cm} \pm 8.6 \mathrm{~cm}$, respectively, $\mathrm{p}=0.80$ ) session, also with small ES (0.19 and 0.01 , respectively).

All RIA measures were similar between sessions except for FI and maximum RPE (Table $3^{19}$ ). Most RIA performance measures exhibited excellent test-retest reliability (ICC $=0.92-0.97$ ), good levels of agreement (ratio LOA $=1.05-1.06$ ) and low measurement error $\left(\mathrm{CV}=2.17-2.68 \%\right.$, Table $\left.3^{19}\right)$. However, FI and average RPE demonstrated moderate test-retest reliability ( $\mathrm{ICC}=0.87$ and 0.76 , respectively), poorer levels of agreement (ratio LOA $=2.57$ and 2.23, respectively) and higher measurement error $(\mathrm{CV}=25.3$ and $15.8 \%$, respectively, Table $3^{19}$ ).

For the RTT, excellent test-retest reliability $(\mathrm{ICC}=0.91)$, good levels of agreement (ratio LOA $=1.08$ ) and low measurement error $(\mathrm{CV}=2.17-2.68 \%)$ were identified for a few variables $\left(\right.$ Table $\left.3^{19}\right)$. However, high test-retest reliability $($ ICC $=$ 0.93), lower levels of agreement (ratio LOA $=1.78$ ) and higher levels of measurement error $(\mathrm{CV}=12.3 \%)$ were observed for

Table 1. Relationship between performance measures of the repeated Illinois agility test (RIA), repeated T-agility test (RTT) and repeated sprint ability (RSA).

\begin{tabular}{|c|c|c|c|c|c|c|c|c|}
\hline & \multicolumn{4}{|c|}{ RIA } & \multicolumn{4}{|c|}{ RSA } \\
\hline & TT (s) & BT (s) & AT (s) & FI (\%) & TT (s) & BT (s) & AT (s) & FI (\%) \\
\hline \multicolumn{9}{|l|}{ RTT } \\
\hline $\mathrm{TT}(\mathrm{s})$ & $0.84^{\star * *}$ & $0.81^{* * *}$ & $0.84^{* * *}$ & $0.51^{\star * *}$ & $0.70^{\text {** }}$ & $0.51^{*}$ & $0.70^{\star *}$ & $0.55^{\text {** }}$ \\
\hline BT (s) & $0.81^{* * *}$ & $0.81^{* * *}$ & $0.81^{* * *}$ & $0.44^{* * *}$ & $0.71^{\text {** }}$ & $0.54^{*}$ & $0.71^{* *}$ & $0.50^{*}$ \\
\hline AT (s) & $0.84^{\star \star *}$ & $0.80^{* * *}$ & $0.84^{\star * *}$ & $0.51^{\star * *}$ & $0.70^{\star *}$ & $0.51^{*}$ & $0.70^{* *}$ & $0.55^{\text {** }}$ \\
\hline $\mathrm{FI}(\%)$ & $0.43^{\star *}$ & 0.32 & $0.43^{* *}$ & $0.48^{\star *}$ & 0.28 & 0.13 & 0.28 & 0.37 \\
\hline \multicolumn{9}{|l|}{ RSA } \\
\hline $\mathrm{TT}(\mathrm{s})$ & $0.80^{\star * *}$ & $0.73^{\text {***}}$ & $0.80^{* * *}$ & $0.55^{* * *}$ & - & - & - & - \\
\hline BT (s) & $0.63^{\star * *}$ & $0.51^{* * *}$ & $0.63^{* * *}$ & $0.56^{* * *}$ & - & - & - & - \\
\hline AT (s) & $0.80^{* * *}$ & $0.73^{* * *}$ & $0.80^{* * *}$ & $0.55^{\star * \star}$ & - & - & - & - \\
\hline $\mathrm{FI}(\%)$ & $0.48^{* *}$ & $0.61^{* * *}$ & $0.49^{* *}$ & 0.28 & - & - & - & - \\
\hline
\end{tabular}

$\mathrm{TT}=$ total time; $\mathrm{BT}=$ best time; $\mathrm{AT}=$ average time; $\mathrm{FI}=$ fatigue index

${ }^{*} \mathrm{P}<0.05{ }^{* *} \mathrm{P}<0.01{ }^{* * *} \mathrm{P}<0.001$ 


\begin{abstract}
Table 2. Pearson correlation coefficients between repeated performances, perceptual and physiological indices (repeated Illinois agility [RIA], repeated T-agility test [RTT]) with aerobic capacity, leg power, speed, and agility test performance measures.
\end{abstract}

\begin{tabular}{|c|c|c|c|c|c|}
\hline & $\begin{array}{c}\mathrm{VO}_{2 \max } \\
\left(\mathrm{mL} \cdot \mathrm{kg}^{-1} \cdot \mathrm{min}^{-1}\right)\end{array}$ & CMJ (cm) & Sprint $30 \mathrm{~m}$ (sec) & IA (sec) & TTA (sec) \\
\hline \multicolumn{6}{|l|}{ RIA } \\
\hline $\mathrm{TT}(\mathrm{s})$ & $-0.73^{* *}$ & $-0.85^{\star *}$ & $0.89^{* *}$ & $0.87^{* *}$ & $0.72^{* *}$ \\
\hline $\mathrm{BT}(\mathrm{s})$ & $-0.71^{* *}$ & $-0.79^{* *}$ & $0.81^{* *}$ & $0.86^{\star *}$ & $0.71^{* *}$ \\
\hline AT (s) & $-0.73^{* *}$ & $-0.85^{* *}$ & $0.89^{* *}$ & $0.87^{\star *}$ & $0.72^{* *}$ \\
\hline $\mathrm{FI}(\%)$ & $-0.43^{*}$ & $-0.57^{\star *}$ & $0.61^{* *}$ & $0.49^{*}$ & 0.40 \\
\hline $\mathrm{HR}_{\text {Avg }}$ & -0.43 & -0.09 & 0.34 & 0.24 & 0.38 \\
\hline $\mathrm{HR}_{\text {Max }}$ & -0.20 & -0.03 & 0.12 & 0.04 & 0.21 \\
\hline $\mathrm{RPE}_{\text {Avg }}$ & 0.17 & 0.17 & -0.21 & -0.16 & -0.21 \\
\hline $\mathrm{RPE}_{\mathrm{Max}}$ & -0.04 & -0.23 & 0.27 & 0.35 & 0.21 \\
\hline \multicolumn{6}{|l|}{$\mathrm{RTT}$} \\
\hline $\mathrm{TT}(\mathrm{s})$ & $-0.68^{* *}$ & $-0.76^{* *}$ & $0.80^{\star *}$ & $0.84^{* *}$ & $0.80^{* *}$ \\
\hline BT (s) & $-0.65^{\text {** }}$ & $-0.74^{* *}$ & $0.80^{* *}$ & $0.86^{* *}$ & $0.85^{\star *}$ \\
\hline AT $(s)$ & $-0.68^{* *}$ & $-0.76^{* *}$ & $0.80^{* *}$ & $0.84^{* *}$ & $0.80^{* *}$ \\
\hline $\mathrm{FI}(\%)$ & -0.41 & -0.37 & 0.34 & 0.28 & 0.14 \\
\hline $\mathrm{HR}_{\text {Avg }}$ & -0.41 & -0.05 & 0.26 & 0.13 & 0.23 \\
\hline $\mathrm{HR}_{\mathrm{Max}}$ & -0.33 & -0.68 & 0.21 & 0.06 & 0.24 \\
\hline $\mathrm{RPE}_{\text {Avg }}$ & 0.00 & 0.02 & -0.03 & 0.02 & -0.07 \\
\hline $\mathrm{RPE}_{\mathrm{Max}}$ & 0.02 & 0.04 & -0.03 & 0.03 & -0.03 \\
\hline
\end{tabular}

$\mathrm{CMJ}=$ countermovement jump; $\mathrm{TT}=$ total time; $\mathrm{BT}=$ best time; $\mathrm{AT}=$ average time; $\mathrm{FI}=$ fatigue index ; Sprint 30m = 30 metre sprint; IA = Illinois Agility test; TTA = T-test agility; $\mathrm{VO}_{2 \max }=$ maximal aerobic capacity, $\mathrm{RPE}_{\mathrm{Avg}}=$ Average Rate of Perceived Exertion, $\mathrm{RPE}_{\mathrm{Max}}=$ Maximum Rate of Perceived Exertion, $\mathrm{HR}_{\text {Avg }}$ = Heart rate average, $\mathrm{HR}_{\text {Max }}=$ Maximum heart rate ${ }^{*} \mathrm{P}<0.05{ }^{* *} \mathrm{P}<0.01$ ${ }^{* * *} \mathrm{P}<0.001$

maximum RPE (Table $3^{19}$ ). In addition, FI, average RPE and maximum HR displayed moderate to large reliability $(\mathrm{ICC}=$ $0.69-0.89$ ), poorer agreement (ratio LOA $=1.10-2.59$ ) and higher measurement error $(\mathrm{CV}=2.38-27.6 \%)$ compared to the RIA protocol (Table $3^{19}$ ).

\section{Discussion}

The current findings demonstrated strong correlations between the RIA and RTT protocols, specifically the BT, TT and AT measures. These results highlight that most time-derived measures (i.e., BT, TT and AT measures) of the RIA are replicable to a previously established repeated agility protocol, but with movement demands more representative of RL. In addition, the TT and BT of the RIA was strongly associated with the TT and BT of the RSA, indicating that the ability to maintain linear speed would result in superior performances in the RIA protocol, possibly due to similar metabolic demands ${ }^{7}$. Comparable findings were reported by Fessi, Makni ${ }^{9}$, with strong correlations identified between the BT and TT of their repeated agility protocol and RSA protocols in 45 team-sport athletes. Collectively, our results and others ${ }^{7,9}$, suggest that performance of repeated agility relies heavily upon the anaerobic system, a metabolic pathway predominant in $\mathrm{RL}^{20}$.

The current study also identified strong test-retest reliability for time-derived measures (i.e., BT, TT and AT) of the RIA, with minimal measurement error. However, the measurement error was substantially higher for FI, confirming previous studies that reported substantially stronger reliability measures for BT, TT and AT compared to that of FI from various repeated agility protocols ${ }^{7,8,21}$. It has been suggested that FI may exhibit weaker reproducibility as the measure is multifactorial and dependent on the stability of other variables (i.e., TT and BT $)^{7,21}$. Subsequently, we, and others ${ }^{7,8,21,22}$, recommend that time-derived measures be primarily evaluated during repeated agility protocols.

Another novelty of the current study was the reliability of the psychophysiological responses during both RIA and RTT protocols. The test-retest reliability values for HR and RPE ranged between questionable-to-excellent classifications according to ICC scores for both RIA and RTT. However, distinctly 


\begin{abstract}
Table 3. Test-retest results, intra-class correlation coefficients (ICC, 95\% confidence interval (CI)), measurement bias/ratio (log-transformed data) ( $\times / \div 95 \%$ ratio limits of agreement (ratio-LOA)) and within-subject coefficient of variation $(95 \% \mathrm{Cl})$ of the repeated Illinois Agility (RIA) and T-test (RTT) protocol.
\end{abstract}

\begin{tabular}{|c|c|c|c|c|c|c|}
\hline & Test (s) & Retest (s) & p & ICC (95\% CI) & CV\% $(95 \% \mathrm{Cl})$ & Bias ratio-LOA \\
\hline \multicolumn{7}{|l|}{ RIA } \\
\hline $\mathrm{TT}(\mathrm{s})$ & $108.22 \pm 9.14$ & $107.38 \pm 8.39$ & 0.23 & $0.97(0.92-0.99)^{\star \star *}$ & $1.97(0.91-2.16)$ & $1.01 \times / 1.06$ \\
\hline BT (s) & $17.00 \pm 1.03$ & $17.05 \pm 1.05$ & 0.60 & $0.96(0.90-0.98)^{* * *}$ & $1.77(0.98-1.83)$ & $1.00 \times / 1.05$ \\
\hline AT (s) & $18.04 \pm 1.52$ & $17.90 \pm 1.40$ & 0.23 & $0.97(0.92-0.99)^{\star \star *}$ & $1.97(0.91-2.16)$ & $1.01 \times / 1.06$ \\
\hline $\mathrm{FI}(\%)$ & $6.02 \pm 3.50$ & $4.91 \pm 3.18$ & $0.03+$ & $0.87(0.68-0.95)^{\star \star *}$ & $25.3(22.9-40.1)$ & $1.32 \times / 2.57$ \\
\hline $\mathrm{RPE}_{\text {Avg }}$ & $4.9 \pm 1.2$ & $4.3 \pm 1.7$ & 0.07 & $0.76(0.41-0.90)^{* *}$ & $15.8(6.1-25.6)$ & $1.20 \times / 2.23$ \\
\hline $\mathrm{RPE}_{\mathrm{Max}}$ & $6.5 \pm 1.6$ & $6.1 \pm 1.7$ & $0.04+$ & $0.93(0.83-0.97)^{\star * *}$ & $8.1(4.8-11.7)$ & $1.08 \times / 1.34$ \\
\hline $\mathrm{HR}_{\text {Avg }}(\mathrm{bpm})$ & $183.8 \pm 8.5$ & $180.2 \pm 10.2$ & 0.09 & $0.92(0.89-0.97)^{\star * *}$ & $2.10(1.48-2.72)$ & $1.02 \times / 1.06$ \\
\hline $\mathrm{HR}_{\mathrm{Max}}(\mathrm{bpm})$ & $189.0 \pm 8.3$ & $188.3 \pm 9.6$ & 0.53 & $0.94(0.83-0.98)^{\star * *}$ & $1.31(0.78-1.84)$ & $1.00 \times / 1.05$ \\
\hline \multicolumn{7}{|l|}{ RTT } \\
\hline $\mathrm{TT}(\mathrm{s})$ & $68.69 \pm 4.79$ & $69.01 \pm 5.15$ & 0.61 & $0.91(0.79-0.96)^{\star \star \star}$ & $2.68(1.91-1.39)$ & $1.00 \times / 1.08$ \\
\hline $\mathrm{BT}(\mathrm{s})$ & $11.01 \pm 0.7$ & $11.06 \pm 0.74$ & 0.58 & $0.91(0.78-0.96)^{\star \star *}$ & $2.17(1.55-2.80)$ & $1.00 \times / 1.08$ \\
\hline AT (s) & $11.45 \pm 0.80$ & $11.50 \pm 0.86$ & 0.61 & $0.91(0.79-0.96)^{\star * *}$ & $2.68(1.91-3.14)$ & $1.00 \times / 1.08$ \\
\hline $\mathrm{FI}(\%)$ & $3.98 \pm 1.68$ & $3.97 \pm 1.89$ & 0.99 & $0.69(0.25-0.87)^{\star * *}$ & $27.6(15.3-33.7)$ & $1.03 \times / 2.59$ \\
\hline $\mathrm{RPE}_{\text {Avg }}$ & $3.2 \pm 1.2$ & $3.6 \pm 1.4$ & 0.10 & $0.89(0.73-0.95)^{\star \star \star}$ & $15.3(8.4-22.2)$ & $0.91 \times / 1.85$ \\
\hline $\mathrm{RPE}_{\text {Max }}$ & $4.4 \pm 1.7$ & $4.9 \pm 1.8$ & $0.02+$ & $0.93(0.84-0.97)^{\star * *}$ & $12.3(4.7-19.8)$ & $0.90 \times / 1.78$ \\
\hline $\mathrm{HR}_{\text {Avg }}(\mathrm{bpm})$ & $176.2 \pm 7.5$ & $174.0 \pm 13.0$ & 0.35 & $0.79(0.45-0.92)^{\star *}$ & $2.88(1.70-4.04)$ & $1.02 \times / 1.11$ \\
\hline $\mathrm{HR}_{\text {Max }}(\mathrm{bpm})$ & $186.3 \pm 8.0$ & $183.7 \pm 10.0$ & 0.20 & $0.68(0.14-0.88)^{\star}$ & $2.38(1.06-3.69)$ & $1.02 \times / 1.10$ \\
\hline
\end{tabular}

$\mathrm{TT}=$ total time; $\mathrm{BT}=$ best time; $\mathrm{AT}=$ average time; $\mathrm{FI}=$ fatigue index, $\mathrm{RPE}_{\text {Avg }}=$ Average Rate of Perceived Exertion, $\mathrm{RPE}_{\mathrm{Max}}=\mathrm{Maximum}_{\mathrm{Rate}}$ of Perceived Exertion, $\mathrm{HR}_{\text {Avg }}=$ Heart rate average, $\mathrm{HR}_{\text {Max }}=$ Maximum heart rate $\mathrm{P}<0.05^{* *} \mathrm{P}<0.01{ }^{* * *} \mathrm{P}<0.001$

† Significantly different $(\mathrm{p}<0.05)$

greater measurement error and bias was observed for RPE when compared to HR measures for both RIA and RTT. These findings were similar to previous studies with poorer reliability for RPE than HR measures during various running protocols ${ }^{23-25}$. It has been postulated that HR has better stability across days given that it is an objective measure, compared to the highly subjective $\mathrm{RPE}^{26}$. It has also been reported that participant's prior knowledge of the number of sprints during repeated sprint-type protocols may affect results due to pacing ${ }^{27}$. Accordingly, HR measures may be a better physiological indicator for monitoring exercise-induced stress during repeated agility protocols.

An additional, yet essential finding of this study was the relationship between baseline characteristics and performances measures from the repeated agility tests. Measures of CMJ, best-effort speed and best-effort agility correlated significantly with the time-derived variables of the RIA. These relationships indicated that lower limb power, linear speed and change-of-direction capabilities were contributing factors to successful repeated agility performances and key attributes needed for RL athletes ${ }^{28}$.
Our findings aligned with those of Haj-Sassi, Dardouri ${ }^{8}$, who reported strong correlations between measures of jump performance and repeated agility performance with an Agility T-test protocol. These authors suggested that larger jumping performances reflected athlete's superior ability to generate force into the ground and therefore a significantly greater change-ofdirection ability ${ }^{8}$. The significance of this finding attests to lower limb power production being a critical component of repeated agility performance, especially within the RIA.

Finally, the current study identified significant correlations between $\mathrm{VO}_{2 \max }$ and RIA performance measures. These findings are similar to previous studies using various repeated agility protocols $^{21,22}$ as well as RSA protocols ${ }^{29-31}$. Measures of $\mathrm{VO}_{2 \max }$ has been considered essential for repeated-sprint type protocols, due to muscular reoxygenation rate ${ }^{8,32}$, optimal capacity to remove and buffer hydrogen ions within working muscles ${ }^{33}$ and efficiently replenish phosphagen stores ${ }^{34}$. The findings of the present study suggest that aerobic capacity is a strong contributor to superior repeated agility efforts, further highlighting 
the need to optimise recovery capacities between high-intensity bouts for RL athletes.

In conclusion, the RIA protocol exhibited moderate-to-excellent test-retest reliability and low measurement error for the majority of time-derived measures and psychophysiological measures, and questionable reliability for FI. Further, this study has clearly demonstrated that repeated agility performances rely upon contributions from both anaerobic and aerobic systems with the RIA, demonstrating that the qualities required for optimal RIA performance may be representative of the physical demands in RL. The RIA protocol may provide practitioners with a simple, yet effective monitoring tool to quantify athlete's ability to generate and sustain multi-directional efforts, and their ability to recover during intermittent activities.

\section{Data availability}

Underlying data

James Cook University Research Data: Convergent validity and reliability of a novel repeated agility protocol in junior rugby league players. https://doi.org/10.25903/5eb0f568fad $20^{29}$

This project contains the following underlying data:

- Raw_data_De-identified.xlsx (Agility protocol data in excel format)

- Raw_data_De-identified.ods (Agility protocol data in ods format)

Data are available under the terms of the Creative Commons Attribution 4.0 International license (CC-BY 4.0).
1. Austin DJ, Gabbett T, Jenkins DJ: Repeated high-intensity exercise in a professional rugby league. J Strength Cond Res. 2011; 25(7): 1898-1904. PubMed Abstract | Publisher Full Text

2. Nimphius S, Callaghan SJ, Bezodis NE, et al:: Change of direction and agility tests: Challenging our current measures of performance. Strength Cond $J$. 2018; 40(1): 26-38. Publisher Full Tex

3. Raya MA, Gailey RS, Gaunaurd IA, et al.: Comparison of three agility tests with male servicemembers: Edgren Side Step Test, T-Test, and Illinois Agility Test. J Rehabil Res Dev. 2013; 50(7): 951-960. PubMed Abstract | Publisher Full Text

4. Durandt J, du Toit S, Borresen J, et al:: Fitness and body composition profiling of elite junior South African rugby players. South Afr J Sports Med. 2006; 18(2) $38-45$. Publisher Full Text

5. Wheeler K: Agility skill execution in rugby union. In: Faculty of Science, Health and Education. University of Sunshine Coast. 2010.

Reference Source

6. King T, Jenkins D, Gabbett T: A time-motion analysis of professional rugby league match-play. J Sports Sci. 2009; 27(3): 213-219. PubMed Abstract | Publisher Full Text

7. Wong DP, Chan GS, Smith AW: Repeated-sprint and change-of-direction abilities in physically active individuals and soccer players: Training and testing implications. J Strength Cond Res. 2012; 26(9): 2324-2330. PubMed Abstract | Publisher Full Text

8. Haj-Sassi R, Dardouri W, Gharbi Z, et al.: Reliability and validity of a new repeated agility test as a measure of anaerobic and explosive power. $J$ Strength Cond Res. 2011; 25(2): 472-480. PubMed Abstract | Publisher Full Text

9. Fessi MS, Makni E, Elloumi M, et al:: Reliability and criterion-related validity of a new repeated agility test. Biol Sport. 2016; 33(2): 159-164. PubMed Abstract | Publisher Full Text | Free Full Text

10. Ramsbottom R, Brewer J, Williams C: A progressive shuttle run test to estimate maximal oxygen uptake. Br J Sports Med. 1988; 22(4): 141-144. PubMed Abstract | Publisher Full Text | Free Full Text

11. Doma K, Nicholls A, Gahreman D, et al.: The effect of a resistance training session on physiological and thermoregulatory measures of sub-maximal running performance in the heat in heat-acclimatized men. Sports Med Open. 2019; 5(1): 21.

PubMed Abstract | Publisher Full Text | Free Full Text

12. Doma K, Schumann M, Leicht AS, et al.: The repeated bout effect of traditional resistance exercises on running performance across 3 bouts. Appl Physiol Nutr Metab. 2017; 42(9): 978-985. PubMed Abstract | Publisher Full Text

13. Ramírez-Campillo R, Meylan C, Alvarez C, et al.: Effects of in-season low-volume high-intensity plyometric training on explosive actions and endurance of young soccer players. J Strength Cond Res. 2014; 28(5): 1335-42. PubMed Abstract | Publisher Full Text

14. Doma K, Schumann M, Sinclair WH, et al:: The repeated bout effect of typical lower body strength training sessions on sub-maximal running performance and hormonal response. Eur J Appl Physiol. 2015; 115(8): 1789-99. PubMed Abstract | Publisher Full Text

15. Zamunér AR, Moreno MA, Camargo TM, et al:: Assessment of subjective perceived exertion at the anaerobic threshold with the borg CR-10 scale. J Sports Sci Med. 2011; 10(1): 130-6. PubMed Abstract | Free Full Text

16. Cordier R, Chen YW, Speyer R, et al:: Child-report measures of occupational performance: a systematic review. PLoS One. 2016; 11(1): e0147751. PubMed Abstract | Publisher Full Text | Free Full Text

17. Leicht AS, Sealey RM, Sinclair $\mathrm{WH}$ : The reliability of $\mathrm{VO}_{2 \text { (peak) }}$ determination in healthy females during an incremental arm ergometry test. Int $J$ Sports Med. 2009; 30(7): 509-15.

PubMed Abstract | Publisher Full Text

18. Nevill AM, Atkinson G: Assessing agreement between measurements recorded on a ratio scale in sports medicine and sports science. Br J Sports Med. 1997; 31(4): 314-8.

PubMed Abstract | Publisher Full Text | Free Full Text

19. Doma K: Convergent validity and reliability of a novel repeated agility protocol in junior rugby league players. James Cook University. (dataset). 2020; 89 b30381 4f7501cfee8dd987fb606ad5

20. Gabbett TJ: Physiological and anthropometric characteristics of junior rugby league players over a competitive season. J Strength Cond Res. 2005; 19(4): 764-771.

PubMed Abstract | Publisher Full Text

21. Zagatto AM, Ardigò LP, Barbieri FA, et al.: Performance and metabolic demand of a new repeated-sprint ability test in basketball players: does the number of changes of direction matter? J Strength Cond Res. 2017; 31(9): 2438-2446. PubMed Abstract | Publisher Full Text

22. Daneshfar A, Gahreman DE, Koozehchian MS, et al:: Multi directional repeated sprint is a valid and reliable test for assessment of junior handball players. Front Physiol. 2018; 9(1): 317

PubMed Abstract | Publisher Full Text | Free Full Text

23. Doma K, Deakin GB, Leicht AS, et al:: The reliability of running economy among trained distance runners and field-based players. J Exerc Sci Phys Fit. 2012; 10(2): 90-96. Publisher Full Text

24. Lamb KL, Eston RG, Corns D: Reliability of ratings of perceived exertion during progressive treadmill exercise. Br J Sports Med. 1999; 33(5): 336-9. PubMed Abstract | Publisher Full Text | Free Full Text

25. Scott TJ, Black CR, Quinn J, et al:: Validity and reliability of the session-RPE method for quantifying training in Australian football: a comparison of the CR10 and CR100 scales. J Strength Cond Res. 2013; 27(1): 270-6. PubMed Abstract | Publisher Full Text

26. Vera J, Perales JC, Jiménez R, et al.: A test-retest assessment of the effects of mental load on ratings of affect, arousal and perceived exertion during submaximal cycling. J Sports Sci. 2018; 36(22): 2521-2530. PubMed Abstract | Publisher Full Text

27. Billaut F, Bishop DJ, Schaerz S, et al.: Influence of knowledge of sprint number 
on pacing during repeated-sprint exercise. Med Sci Sport Exerc. 2011; 43(4): 665-672.

PubMed Abstract | Publisher Full Text

28. Hoffman JR, Epstein S, Einbinder M, et al:: A comparison between the wingate anaerobic power test to both vertical jump and line drill tests in basketball players. J Strength Cond Res. 2000; 14(3): 261-264.

Publisher Full Text

29. Bishop D, Edge J: Determinants of repeated-sprint ability in females matched for single-sprint performance. Eur J Appl Physiol. 2006; 97(4): 373-9.

PubMed Abstract | Publisher Full Text

30. Gharbi Z, Dardouri W, Haj-Sassi R, et al:: Aerobic and anaerobic determinants of repeated sprint ability in team sports athletes. Biol Sport. 2015; 32(3): 207-12.

PubMed Abstract | Publisher Full Text | Free Full Text
31. Glaister M: Multiple sprint work: physiological responses, mechanisms of fatigue and the influence of aerobic fitness. Sports Med. 2005; 35(9): 757-77. PubMed Abstract | Publisher Full Text

32. Buchheit M, Ufland P: Effect of endurance training on performance and muscle reoxygenation rate during repeated-sprint running. Eur J Appl Physiol. 2011 111(2): 293-301.

PubMed Abstract | Publisher Full Text

33. Sahlin K, Henriksson J: Buffer capacity and lactate accumulation in skeletal muscle of trained and untrained men. Acta Physiol Scand. 1984; 122(3): 331-9. PubMed Abstract | Publisher Full Text

34. Bogdanis GC, Nevill ME, Boobis LH, et al:: Contribution of phosphocreatine and aerobic metabolism to energy supply during repeated sprint exercise. $J \mathrm{Appl}$ Physiol (1985). 1996; 80(3): 876-84.

PubMed Abstract | Publisher Full Text 


\section{Open Peer Review}

\section{Current Peer Review Status: ? ?}

\section{Version 1}

Reviewer Report 03 August 2021

https://doi.org/10.5256/f1000research.25532.r90678

(C) 2021 Scanlan A. This is an open access peer review report distributed under the terms of the Creative Commons Attribution License, which permits unrestricted use, distribution, and reproduction in any medium, provided the original work is properly cited.

\section{Aaron T. Scanlan}

Human Exercise and Training Laboratory, School of Health, Medical and Applied Sciences, Central Queensland University, Rockhampton, Qld, Australia

Thank you for the opportunity to review this manuscript. A logical study with potential practical impact was presented exploring the validity and reliability of a repeated change-of-direction speed test suited to rugby league. This aspect of fitness testing is lacking in the literature and of use for end-users, so this manuscript has merit for publication. I do however have some suggestions and queries listed below that will help strengthen some aspects of the work that should be addressed by the authors:

1. Abstract, Methods - indicate whether you are referring to the multi-stage fitness test (i.e., Beep test) here or if just an assessment of multi-stage fitness was performed.

2. Abstract - some justification of using the repeated T-agility test as the other standard repeated change-of-direction performance to assess convergent agility of the repeated Illinois Agility test is needed in the Abstract.

3. Abstract - the focus of exploring the contribution of different fitness attributes to repeated Illinois Agility test performance is not made in the Abstract. Consider including this as a secondary objective and/or making it clear why this analysis is needed.

4. Abstract, Conclusions - make it clear that this test is useful for Rugby League coaches specifically, and change "athlete" to plural form or indicate "team" here instead.

5. Introduction, opening paragraph - in this paragraph you identify "agility" and hint that it involves physical and cognitive components. So in essence, you are examining change-ofdirection speed (the physical component) rather than "agility" per se. Consider making this clear and using the term "change-of-direction speed" or "change-of-direction performance" thereafter when referring to tests and attributes that are purely physical without the cognitive component.

6. Introduction, opening paragraph - stronger rationale is needed justifying the inclusion of 
the Agility T-test and Illinois Agility test in rugby league. Can you add a sentence or two outlining why these tests are suited to the sport and therefore the focus of your study?

7. Introduction, end of second paragraph - here indicate whether the validity and reliability of this test has not been investigated at all or just specifically in Rugby League athletes. Also, at the end of this sentence make it clear that you are referring to the usability of this test.

8. Introduction, aims - for the second aim, you are not comparing the RIA measures to other measures, but instead correlating them, so please change this aim accordingly. Also, consider including a little rationale around this aim in the previous paragraph as it is unclear as to why this is important.

9. Introduction, aims - it might pay to include a sentence stating why examining convergent validity (e.g. to show that you are assessing similar attributes with a new test that is more practical and specific to Rugby League when compared to a standard, generic test routinely used) and retest reliability (e.g. to detect the inherent error in the test and ascertain whether it can be reliably adopted in practice to assess repeated measurements in athletes) are needed for practical uptake of the test, which would strengthen the rationale of the first and third aims as well in the Introduction section.

10. Introduction, hypotheses - you only mention speed and anaerobic capacity as your fitness attributes here, but you also included aerobic fitness (Multistage Fitness test)?

11. Methods, Research design - why was the order flipped between session 4 and session 5 ? It seems like you would want to the athletes to complete the same exact session across both when assessing retest reliability as it is introducing a confounding factor? Also, no hyphen needed between "15" and "minutes" here.

12. Methods, Participants - can you provide some further indication as to the specific playing level of the athletes? The name of the program is great, but this is not exactly clear for all readers.

13. Figure 1 - consider changing the session numbers, as in text you identify the Multistage Fitness test as session 1, but here you indicate the other fitness testing as session 1. Consistency needed.

14. Methods, Participants - add "in" after "partaking" and make parents/guardians singular rather than plural.

15. Methods, Multistage Shuttle test - stay consistent with capitalising the test names like this one as it is done inconsistently throughout. Also, hyphenate " $20 \mathrm{~m}$ " here.

16. Methods, Countermovement jump test - hyphenate " $1 \mathrm{~cm}$ " here. Also, try to indicate what units the key outcomes from each test were reported in (e.g. $\mathrm{mL} / \mathrm{kg} / \mathrm{min}, \mathrm{cm}, \mathrm{s}$ ).

17. Methods, 30-m Sprint and Agility protocols - separate statements on linear and change-ofdirection speed here. Also, hyphenate " $10 \mathrm{~m}$ " and " $5 \mathrm{~m}$ " for the Illinois Agility test. 
18. Methods, repeat protocols - to calculate average HR and RPE, was HR measured from when the test started to when the test finished in 1-second intervals? And was RPE taken after each effort or just after all efforts for each specific test? These are not quite clear.

19. Methods, Statistical analysis - you mention "construct" validity for the first time here. If this is a key aim and aspect of the study (i.e., correlating performance during the test with fitness attributes), then this needs to be established earlier (i.e., introduction and aims).

20. Methods, Statistical analysis - an $r$ value of 0.5 seems quite low to establish convergent validity (only $25 \%$ shared variance)? In this regard, what was the cut-off for construct validity?

21. Results, first paragraph - clarify whether this is CMJ height specifically.

22. Results, second paragraph - make it clear that you are referring to the third and fourth sessions here (and make sure this is consistent as you identify these as the fourth and fifth sessions earlier).

23. Results, third paragraph - you do not indicate the maximum RPE was taken as an outcome in the methods anywhere (only average), yet it is listed here? Same for maximum HR, which appears later in this section also.

24. Table 1 - shouldn't RIA and RTT be the two key tests going from left to right rather than RIA and RSA? In fact, it is not clear why RSA is included here given you identify RIA vs. RTT for convergent validity assessment throughout the manuscript. Please adjust or rework earlier sections.

25. Discussion, first paragraph - some minor errors, but change "was" to "were" in the $7^{\text {th }}$ line.

26. Discussion, first paragraph - at the end of this paragraph, some explanation as to how yours (and the previous studies) support that anaerobic fitness is predominantly stressed in the repeated COD tests. I am assuming the strong correlations with mostly anaerobic fitness attributes, but this is an assumption and should be explained further to clarify this statement.

27. Discussion, fourth paragraph - be careful with your statement suggesting they are "key attributes for RL athletes". You did not show this but state it, so rework or remove this part to focus specifically on what your data show.

28. Discussion, concluding paragraph - here you highlight how anaerobic and aerobic fitness underpin test performance, but previously you focus on anaerobic fitness in other sections and in this section. Please make sure the message is consistent throughout.

\section{Is the work clearly and accurately presented and does it cite the current literature? Yes}

Is the study design appropriate and is the work technically sound? 
Partly

Are sufficient details of methods and analysis provided to allow replication by others? Yes

If applicable, is the statistical analysis and its interpretation appropriate? Yes

Are all the source data underlying the results available to ensure full reproducibility? Yes

Are the conclusions drawn adequately supported by the results?

Yes

Competing Interests: No competing interests were disclosed.

Reviewer Expertise: Exercise and sport science

I confirm that I have read this submission and believe that I have an appropriate level of expertise to confirm that it is of an acceptable scientific standard, however I have significant reservations, as outlined above.

Author Response 27 Sep 2021

Kenji Doma, James Cook University, Douglas, Australia

Reviewer's comment: Thank you for the opportunity to review this manuscript. A logical study with potential practical impact was presented exploring the validity and reliability of a repeated change-of-direction speed test suited to rugby league. This aspect of fitness testing is lacking in the literature and of use for end-users, so this manuscript has merit for publication. I do however have some suggestions and queries listed below that will help strengthen some aspects of the work that should be addressed by the authors:

Author's response: Dear Reviewer, thank you very much for your comments. We believe your feedback has improved the quality of our work.

Reviewer's comment: Abstract, Methods - indicate whether you are referring to the multistage fitness test (i.e., Beep test) here or if just an assessment of multi-stage fitness was performed.

Author's response: A Beep test was performed, which is now included in the Abstract.

Reviewer's comment: Abstract - some justification of using the repeated T-agility test as the other standard repeated change-of-direction performance to assess convergent agility of the repeated Illinois Agility test is needed in the Abstract.

Author's response: This is a valid point. The Background now includes, "Thus, the current study examined the convergent validity of a repeated Illinois Agility (RIA) protocol with the repeated T-agility protocol, and the repeatability of the RIA protocol in adolescent Rugby League players." 
Reviewer's comment: Abstract - the focus of exploring the contribution of different fitness attributes to repeated Illinois Agility test performance is not made in the Abstract. Consider including this as a secondary objective and/or making it clear why this analysis is needed.

Author's response: Thank you for the suggestion. We have now included, "Furthermore, aerobic capacity and anaerobic and COD performance were assessed to determine whether these physical qualities were important contributors to the RIA protocol."

Reviewer's comment: Abstract, Conclusions - make it clear that this test is useful for Rugby League coaches specifically, and change "athlete" to plural form or indicate "team" here instead.

Author's response: The conclusion has been rewritten to, "The current study has demonstrated the RIA to be a simple, valid and reliable field test for RL athletes that can provide coaches with information about their team's ability to sustain high intensity, multi-directional running efforts."

Reviewer's comment: Introduction, opening paragraph - in this paragraph you identify "agility" and hint that it involves physical and cognitive components. So in essence, you are examining change-of-direction speed (the physical component) rather than "agility" per se. Consider making this clear and using the term "change-of-direction speed" or "change-ofdirection performance" thereafter when referring to tests and attributes that are purely physical without the cognitive component.

Author's response: All the terms with 'agility' has been replaced with 'change-ofdirection' performance or protocols throughout the text.

Reviewer's comment: Introduction, opening paragraph - stronger rationale is needed justifying the inclusion of the Agility T-test and Illinois Agility test in rugby league. Can you add a sentence or two outlining why these tests are suited to the sport and therefore the focus of your study?

Author's response: We have removed Agility T-test and Illinois Agility test from the first paragraph, and focused the justification of the Illinois Agility test for RL in the second paragraph (see lines 41-47).

Reviewer's comment: Introduction, end of second paragraph - here indicate whether the validity and reliability of this test has not been investigated at all or just specifically in Rugby League athletes. Also, at the end of this sentence make it clear that you are referring to the usability of this test.

Author's response: We separated the second paragraph into two paragraphs. Thus, we highlighted that no studies have examined the validity and reliability of the RIA protocol at the end of the fourth paragraph.

Reviewer's comment: Introduction, aims - for the second aim, you are not comparing the RIA measures to other measures, but instead correlating them, so please change this aim accordingly. Also, consider including a little rationale around this aim in the previous paragraph as it is unclear as to why this is important.

Author's response: Changed to 'correlating' as requested. The previous paragraph expanded on the need to examine the validity of the RIA protocol, which we now hope 
provides further justification of our aims.

Reviewer's comment: Introduction, aims - it might pay to include a sentence stating why examining convergent validity (e.g. to show that you are assessing similar attributes with a new test that is more practical and specific to Rugby League when compared to a standard, generic test routinely used) and retest reliability (e.g. to detect the inherent error in the test and ascertain whether it can be reliably adopted in practice to assess repeated measurements in athletes) are needed for practical uptake of the test, which would strengthen the rationale of the first and third aims as well in the Introduction section.

Author's response: This is a great suggestion. We have now worded the end of the Introduction to, "Examining the convergent validity of the RIA protocol will determine whether this novel assessment exhibits similar attributes to a standardised COD protocol (i.e., RTT). In addition, the reliability of the RIA will determine whether this test can be reliably adopted in practice by accounting for the inherent error of the test across repeated measurements. The quality of these psychometric properties will provide coaches with a tool to assist in monitoring and training $R L$ athletes as well as in talent development and identification."

Reviewer's comment: Introduction, hypotheses - you only mention speed and anaerobic capacity as your fitness attributes here, but you also included aerobic fitness (Multistage Fitness test)?

Author's response: Included aerobic capacity in the hypotheses.

Reviewer's comment: Methods, Research design - why was the order flipped between session 4 and session 5 ? It seems like you would want to the athletes to complete the same exact session across both when assessing retest reliability as it is introducing a confounding factor? Also, no hyphen needed between "15" and "minutes" here.

Author's response: This is a good question. We employed a cross-over design for sessions 4 and 5 to limit the confounder of any learning effects (i.e., were participants still able to exhibit reproducible measures when the protocols were swapped around, as opposed to the participants becoming accustomed to the order effects between sessions). We have also included the hyphen.

Reviewer's comment: Methods, Participants - can you provide some further indication as to the specific playing level of the athletes? The name of the program is great, but this is not exactly clear for all readers.

Author's response: Further description of the excellence program has been provided.

Reviewer's comment: Figure 1 - consider changing the session numbers, as in text you identify the Multistage Fitness test as session 1, but here you indicate the other fitness testing as session 1. Consistency needed.

Author's response: Session 1 included in the Multistage Fitness test as requested (see updated Figure 1).

Reviewer's comment: Methods, Participants - add "in" after "partaking" and make parents/guardians singular rather than plural.

Author's response: Amended as requested. 
Reviewer's comment: Methods, Multistage Shuttle test - stay consistent with capitalising the test names like this one as it is done inconsistently throughout. Also, hyphenate " $20 \mathrm{~m}$ " here. Author's response: Amended the test name to upper case as requested and hyphenated to $20-\mathrm{m}$.

Reviewer's comment: Methods, Countermovement jump test - hyphenate " $1 \mathrm{~cm}$ " here. Also, try to indicate what units the key outcomes from each test were reported in (e.g. $\mathrm{mL} / \mathrm{kg} / \mathrm{min}, \mathrm{cm}, \mathrm{s})$.

Author's response: Hyphenated to 1-cm and reported units of measure as requested for each test.

Reviewer's comment: Methods, 30-m Sprint and Agility protocols - separate statements on linear and change-of-direction speed here. Also, hyphenate " $10 \mathrm{~m}$ " and " $5 \mathrm{~m}$ " for the Illinois Agility test.

Author's response: Statements have been separated and hyphenated as requested.

Reviewer's comment: Methods, repeat protocols - to calculate average HR and RPE, was HR measured from when the test started to when the test finished in 1-second intervals? And was RPE taken after each effort or just after all efforts for each specific test? These are not quite clear.

Author's response: We have included “ "The participant's instantaneous heart rate (HR, Polar Heart Rate Monitor, Polar H10, Finland) and rating of perceived-exertion (RPE, Borg category scale 1-10) were collected at the completion of each repetition of the RSA, RTT and RIA protocols. The maximum and average HR and RPE values were then reported from the 6 repetitions. - see lines 138-141.

Reviewer's comment: Methods, Statistical analysis - you mention "construct" validity for the first time here. If this is a key aim and aspect of the study (i.e., correlating performance during the test with fitness attributes), then this needs to be established earlier (i.e., introduction and aims).

Author's response: We have taken out "construct validity" and mentioned aerobic capacity, power and COD as "contributors to the RIA protocol".

Reviewer's comment: Methods, Statistical analysis - an $r$ value of 0.5 seems quite low to establish convergent validity (only $25 \%$ shared variance)? In this regard, what was the cutoff for construct validity?

Author's response: This has been re-established to a minimum r-value of 0.7 for both convergent validity and contributors to the RIA protocol.

Reviewer's comment: Results, first paragraph - clarify whether this is CMJ height specifically. Author's response: Height included as requested.

Reviewer's comment: Results, second paragraph - make it clear that you are referring to the third and fourth sessions here (and make sure this is consistent as you identify these as the fourth and fifth sessions earlier).

Author's response: Sessions numbers included as requested, and we also applied this 
approach in the next paragraph for the RTT protocol to ensure clarity.

Reviewer's comment: Results, third paragraph - you do not indicate the maximum RPE was taken as an outcome in the methods anywhere (only average), yet it is listed here? Same for maximum HR, which appears later in this section also.

Author's response: This is a great pick up. In the methods section, we have now included, "The participant's heart rate (HR, Polar Heart Rate Monitor, Polar H10, Finland) and rating of perceived-exertion (RPE, Borg category scale 1-10) were collected at the completion of each repetition of the RSA, RTT and RIA protocols. The maximum and average HR and RPE values were then reported from the 6 repetitions."

Reviewer's comment: Table 1 - shouldn't RIA and RTT be the two key tests going from left to right rather than RIA and RSA? In fact, it is not clear why RSA is included here given you identify RIA vs. RTT for convergent validity assessment throughout the manuscript. Please adjust or rework earlier sections.

Author's response: Table 1 has now been amended as requested. RSA was included more to determine the importance of the athlete's ability to sustain speed and effort over repeated bouts, which has now been included in the second aim.

Reviewer's comment: Discussion, first paragraph - some minor errors, but change "was" to "were" in the 7th line.

Author's response: Amended as requested.

Reviewer's comment: Discussion, first paragraph - at the end of this paragraph, some explanation as to how yours (and the previous studies) support that anaerobic fitness is predominantly stressed in the repeated COD tests. I am assuming the strong correlations with mostly anaerobic fitness attributes, but this is an assumption and should be explained further to clarify this statement.

Author's response: Closer to the end of this paragraph, we have included a sentence that reads, "The comparable measures between RIA, RTT and RSA suggests that anaerobic fitness, in conjunction with efficient recovery dynamics during short periods of rest in-between explosive activities, are essential qualities for optimal performance in an RIA protocol."

Reviewer's comment: Discussion, fourth paragraph - be careful with your statement suggesting they are "key attributes for RL athletes". You did not show this but state it, so rework or remove this part to focus specifically on what your data show.

Author's response: Removed as requested.

Reviewer's comment: Discussion, concluding paragraph - here you highlight how anaerobic and aerobic fitness underpin test performance, but previously you focus on anaerobic fitness in other sections and in this section. Please make sure the message is consistent throughout.

Author's response: We have now included aerobic capacity as possible important contributors to the RIA protocol in the Abstract, Introduction (aim and hypothesis) and earlier sections in the Discussion. 
Competing Interests: No competing interests were disclosed.

Reviewer Report 09 November 2020

https://doi.org/10.5256/f1000research.25532.r74005

(c) 2020 Dugdale J. This is an open access peer review report distributed under the terms of the Creative Commons Attribution License, which permits unrestricted use, distribution, and reproduction in any medium, provided the original work is properly cited.

\author{
James Dugdale \\ 1 School of Applied Sciences, University of Stirling, Stirling, UK \\ 2 Edinburgh Napier University, Edinburgh, UK
}

The present study aimed to explore the validity and reliability of repeated "agility" tests in a sample of youth rugby players. The study provides interesting data for an applied audience and is methodologically sound and accurate in the presentation of findings. This being said, I have identified a range of areas that should be improved prior to potential resubmissions of this manuscript. For example, the introduction (and study in its entirety) is inaccurate in its use of the term "agility" (opposed to COD which the authors actually assess). Furthermore, the introduction needs to be further developed to support the scope of the study. The authors need to create more of a rationale for running such a large number of correlations - exploring correlational analyses needs to be warranted based upon an assumption of a relationship (or lack thereof) between variables. In its current form, I am unsure whether this manuscript provides such rationale. The methods and results are accurate, but with a large number of small errors. Finally, the discussion addresses key discussion points formulated from the findings of the study, but similar to the introduction, lacks some critical depth.

I do think that the authors have a publishable study here, however, a number of revisions will be required before I would be happy to approve this manuscript for indexing.

I hope that my comments are received as I intend them and are useful in further strengthening this manuscript. I hope to receive the opportunity to review this manuscript again in its revised form.

\title{
Abstract
}

The abstract is concise and accurate to the details of the study. I have just a couple of minor comments (below):

The initial sentence is accurate and outlines the requirement of the study. However, I would encourage the authors to insert a word such as 'simultaneously' or 'congruently', or attempt to capture the lack of a suitable test to capture the performance of these abilities in combination. Abstract Methods - the inclusion of the repeated T-agility test appears suddenly with no prior reference. If this is a key concentration of your study, I would encourage to insert earlier in the abstract along with the RIA.

Abstract Results - please specify exact $p$ values rather than just $<0.05$. CV\% can be presented to 
$1 \mathrm{dp}$ for brevity.

\section{Introduction}

The introduction is short and concise. However, I feel some key information that would further support the rationale for your study has been omitted. I would encourage the authors to further develop this section. A suggestion would be to expand the second paragraph into two: the first discussing the validity aspects, the second the reliability aspects. I do not expect these changes to be too onerous, but believe it would strengthen this section of your manuscript if you were to implement these changes. I have some further specific comments below.

The opening sentence would read better if it were split into two separate sentences. The authors should attempt to clarify during opening paragraph that they are discussing change of direction (COD) performance, rather than agility. Although COD performance is a key component of agility performance, the lack of an external stimuli prevents classification of agility. Although the tests identified by the authors in this opening paragraph have titled themselves 'agility' tests, by definition they are not. The authors should be careful in their stance here as the literature has evolved substantially since the inception of tests such as these, and appropriate classification and terminology is essential for the present study.

Young, W.; Dawson, B.; Henry, G. Agility and Change-of-Direction Speed are Independent Skills: Implications for Agility in Invasion Sports. Int. J. Sports Sci. Coach. 2015, 10, 159-169. ${ }^{1}$ Nimphius, S.; Callaghan, S.J.; Bezodis, N.E.; Lockie, R.G. Change of Direction and Agility Tests: Challenging Our Current Measures of Performance. Strength Cond. J. 2018, 40, 26-38. 2 Sheppard, J.M.; Young, W.B. Agility literature review: Classifications, training and testing. J. Sports Sci. 2006, 24, 919-932. ${ }^{3}$

Regarding the reliability aspect of the study, two recent papers have suggested that reliability of COD and agility may be lower in adolescent and youth athletes (as per your sample). Although these authors observed this during maximal COD/agility performance, opposed to repeated as in the present study, I believe this would be worth including and would further warrant the exploration of your research question and study.

Taylor, J.M.; Cunningham, L.; Hood, P.; Thorne, B.; Irvin, G.; Weston, M. The reliability of a modified 505 test and change-of-direction deficit time in elite youth football players. Sci. Med. Footb. 2019, 3, 157-162. ${ }^{4}$

Dugdale JH, Sanders D, Hunter AM. Reliability of Change of Direction and Agility Assessments in Youth Soccer Players. Sports (Basel). 2020 Apr 18; 8 (4): 51. doi: 10.3390/sports8040051. ${ }^{5}$ James H. Dugdale, Calum A. Arthur, Dajo Sanders \& Angus M. Hunter (2019) Reliability and validity of field-based fitness tests in youth soccer players, European Journal of Sport Science, 19:6, 745756, DOI: 10.1080/17461391.2018.15567396

\section{Methods}

The methods report the appropriate details relating to the study design, protocols, participants, and analyses performed. However, further clarity is required in a few places throughout this section (specific comments below). Further, there are a number of oversights throughout this section that require careful attention. None of these are major points, and should take little time to amend. Yet, need to be addressed prior to potential resubmissions of this manuscript. Figure 1: I am unsure how much this figure aids the interpretation of the study design. I personally do not feel the visual nature of this schematic clarifies the description of the trials from the text, as the study is relatively simplistic. I do not insist that the authors remove this figure, however, please consider my suggestion that it may not add value to the manuscript and may just be a "figure for the sake of a figure". Secondly, between the trials, the authors have stated that 
washout periods of $\geq 7$ days were implemented. The authors should consider including the range of days (e.g. 7-14 days), as this would provide further information to the reader and allow for more accurate interpretation of the results.

Can a citation or elaboration be provided to rationalise the $\geq 15$ min rest interval between attempts?

The term 'respectively' needs to be included after the explanation of the 1-10 muscle rating scale. Also, a brief sentence as to why this was collected should accompany this methodological point. The authors state that the warm up was the same prior to each session. I would encourage using the term 'standardised' when describing this in their methods to add certainty around their controls. Also, a citation needs to accompany the statement that the CMJ test was implemented to assess recovery. If this is what was intended by the insertion of citation 12, I would encourage moving this citation to the end of the sentence.

Participant details - participant height needs to be reported to $2 \mathrm{dp}$. Can the authors further elaborate on what the "School of Athletic Excellence" is? For example, what standard is this, is it affiliated to a specific school, or region performance programme? This may not be clear to readers.

Multistage shuttle test - a brief sentence stating how performance or a score was derived is necessary (i.e. maximum number of shuttles completed, level, etc.). This information is currently not evident.

CMJ - please state the unit of measurement for this test, and to what degree (e.g. recorded in $\mathrm{cm}$ to the nearest $0.1 \mathrm{~cm}$ ).

30-m sprint and agility protocols - please separate each test into a separate sentence. At present, they appear too cramped together and it is difficult to read (particularly with reference to figures and citations within). Also, please provide citations for the original studies for the T-drill and Illinois agility tests. These need to be credited within the manuscript.

Repeated sprint and agility protocols - were these tests performed one after the other (what I interpret from the term 'cycles') or were they performed independently? Clarity is required to ensure the reader understands which of these interpretations is correct.

The sentence starting "Immediately after each repeated agility cycle..." does not make sense. Please rephrase this.

Statistical analysis - Reference 16 seems a weak reference for this point. I encourage the authors to consider a more suitable citation for selecting this acceptable correlation cut off point.

\section{Results}

The section of the results dedicated to the muscle soreness and CMJ differences (or lack thereof) should be condensed to a singular sentence and placed at the foot of the results section. This is a methodological control and not a key finding from your study, therefore, it should not feature so early and heavily within your results section. Stating that there were no differences, providing ES, and significance values would suffice.

Table 3 - I am interested in your rationale for not providing ES to demonstrate differences (or lack thereof) for your test-retest data? This would add value in my opinion. I would also encourage the authors to present CV\% data to $1 \mathrm{dp}$ both within this table and throughout the manuscript. I notice that the authors use terminology such as "excellent" or "moderate" test-retest reliability, yet they have not included these threshold within the statistical analysis section of the methods. Please include this detail so that the reader understands your criteria.

I also note that the authors say "most RIA performance measures exhibited excellent test-retest reliability...". Could the authors rephrase this to say "all RIA performance measures except... measures exhibited excellent test-retest reliability"? This would be more accurate and less 
ambiguous for the reader. This comment also applies later in this section where the authors refer to "a few variables...".

\section{Discussion}

I found the discussion well-written and thought the authors accurately interpreted and attempted to explain their findings. However, similar to the introduction, I felt this section lacked depth when discussing the main findings of the study. I encourage the authors to discuss their findings in greater depth, utilising some of the reading attached to this review.

I encourage the authors to utilise the first paragraph of the discussion to summarise all of their main findings, and then progress to discussing each point in turn.

The third paragraph begins "another novelty of this study", yet this appears to be the first time novelty is addressed in the discussion?

The fourth paragraph can draw upon a wealth of correlational studies to further support the findings observed here.

Young WB, Dawson B, Henry GJ. Agility and Change-of-Direction Speed are Independent Skills: Implications for Training for Agility in Invasion Sports. International Journal of Sports Science \& Coaching. 2015;10(1):159-169. doi:10.1260/1747-9541.10.1.1591

Spiteri T, Nimphius S, Hart NH, Specos C, Sheppard JM, Newton RU. Contribution of strength characteristics to change of direction and agility performance in female basketball athletes. J Strength Cond Res. 2014 Sep;28(9):2415-23. doi: 10.1519/JSC.0000000000000547. PMID: 24875426. 7

Gabbett, T. J., Kelly, J. N., \& Sheppard, J. M. (2008). Speed, Change of Direction Speed, and Reactive Agility of Rugby League Players. The Journal of Strength \& Conditioning Research, 22, 174-181. http://dx.doi.org/10.1519/JSC.0b013e31815ef7008

Sheppard, J.M.; Young, W.B.; Doyle, T.L.A.; Sheppard, T.A.; Newton, R.U. An evaluation of a new test of reactive agility and its relationship to sprint speed and change of direction speed. J. Sci Med. Sport 2006, 9, 342-349. ${ }^{9}$

The conclusion should be reframed around the key objectives of the study (i.e. that it is reliable and valid). The mention of aerobic and anaerobic energy systems seems misplaced here.

\section{References}

While a variety of key texts are identified and listed within the bibliography and cited throughout the manuscript, a number of key texts are omitted. I encourage the authors to further familiarise themselves with relevant studies assessing reliability and validity of COD and agility in team sport athletes, as well as the texts identified earlier within this review.

Dos'Santos, T.; Thomas, C.; Jones, P.A.; Comfort, P. Mechanical Determinants of Faster Change of Direction Speed Performance in Male Athletes. J. Strength Cond. Res. 2017, 31, 696-705. ${ }^{10}$ Gabbett, T.J.; Kelly, J.N.; Sheppard, J.M. Speed, change of direction speed, and reactive agility of rugby league players. J. Strength Cond. Res. 2008, 22, 174-181. ${ }^{8}$

Oliver, J.L.; Meyers, R.W. Reliability and generality of measures of acceleration, planned agility, and reactive agility. Int. J. Sports Physiol. Perform. 2009, 4, 345-354. ${ }^{11}$

\section{References}

1. Young W, Dawson B, Henry G: Agility and Change-of-Direction Speed are Independent Skills: Implications for Training for Agility in Invasion Sports. International Journal of Sports Science \& Coaching. 2015; 10 (1): 159-169 Publisher Full Text

2. Nimphius S, Callaghan S, Bezodis N, Lockie R: Change of Direction and Agility Tests. Strength and Conditioning Journal. 2018; 40 (1): 26-38 Publisher Full Text 
3. Sheppard JM, Young WB: Agility literature review: classifications, training and testing.J Sports Sci. 2006; 24 (9): 919-32 PubMed Abstract | Publisher Full Text

4. Taylor J, Cunningham L, Hood P, Thorne B, et al.: The reliability of a modified 505 test and change-of-direction deficit time in elite youth football players. Science and Medicine in Football. 2019; 3 (2): 157-162 Publisher Full Text

5. Dugdale JH, Sanders D, Hunter AM: Reliability of Change of Direction and Agility Assessments in Youth Soccer Players.Sports (Basel). 2020; 8 (4). PubMed Abstract | Publisher Full Text

6. Dugdale JH, Arthur CA, Sanders D, Hunter AM: Reliability and validity of field-based fitness tests in youth soccer players.Eur J Sport Sci. 2019; 19 (6): 745-756 PubMed Abstract | Publisher Full Text 7. Spiteri T, Nimphius S, Hart NH, Specos C, et al.: Contribution of strength characteristics to change of direction and agility performance in female basketball athletes.J Strength Cond Res. 2014; 28 (9): 2415-23 PubMed Abstract | Publisher Full Text

8. Gabbett TJ, Kelly JN, Sheppard JM: Speed, change of direction speed, and reactive agility of rugby league players.J Strength Cond Res. 2008; 22 (1): 174-81 PubMed Abstract | Publisher Full Text

9. Sheppard JM, Young WB, Doyle TL, Sheppard TA, et al.: An evaluation of a new test of reactive agility and its relationship to sprint speed and change of direction speed.J Sci Med Sport. 2006; 9

(4): 342-9 PubMed Abstract | Publisher Full Text

10. Dos'Santos T, Thomas C, Jones PA, Comfort P: Mechanical Determinants of Faster Change of Direction Speed Performance in Male Athletes.J Strength Cond Res. 2017; 31 (3): 696-705 PubMed Abstract | Publisher Full Text

11. Oliver J, Meyers R: Reliability and Generality of Measures of Acceleration, Planned Agility, and Reactive Agility. International Journal of Sports Physiology and Performance. 2009; 4 (3): 345-354

Publisher Full Text

Is the work clearly and accurately presented and does it cite the current literature? Partly

Is the study design appropriate and is the work technically sound?

Yes

Are sufficient details of methods and analysis provided to allow replication by others? Yes

If applicable, is the statistical analysis and its interpretation appropriate? Yes

Are all the source data underlying the results available to ensure full reproducibility? Yes

Are the conclusions drawn adequately supported by the results?

Yes

Competing Interests: No competing interests were disclosed.

Reviewer Expertise: Fitness testing; youth athletes; talent identification; talent development; physical performance; strength and conditioning 
I confirm that I have read this submission and believe that I have an appropriate level of expertise to confirm that it is of an acceptable scientific standard, however I have significant reservations, as outlined above.

Author Response 27 Sep 2021

Kenji Doma, James Cook University, Douglas, Australia

Reviewer's comment: The present study aimed to explore the validity and reliability of repeated "agility" tests in a sample of youth rugby players. The study provides interesting data for an applied audience and is methodologically sound and accurate in the presentation of findings. This being said, I have identified a range of areas that should be improved prior to potential resubmissions of this manuscript. For example, the introduction (and study in its entirety) is inaccurate in its use of the term "agility" (opposed to COD which the authors actually assess). Furthermore, the introduction needs to be further developed to support the scope of the study. The authors need to create more of a rationale for running such a large number of correlations - exploring correlational analyses needs to be warranted based upon an assumption of a relationship (or lack thereof) between variables. In its current form, I am unsure whether this manuscript provides such rationale. The methods and results are accurate, but with a large number of small errors. Finally, the discussion addresses key discussion points formulated from the findings of the study, but similar to the introduction, lacks some critical depth. I do think that the authors have a publishable study here, however, a number of revisions will be required before I would be happy to approve this manuscript for indexing. I hope that my comments are received as I intend them and are useful in further strengthening this manuscript. I hope to receive the opportunity to review this manuscript again in its revised form.

Author's response: Dear reviewer, thank you for your comments on the paper. We agree that your comments have improved the quality of our work.

Abstract

Reviewer's comment: The abstract is concise and accurate to the details of the study. I have just a couple of minor comments (below):

The initial sentence is accurate and outlines the requirement of the study. However, I would encourage the authors to insert a word such as 'simultaneously' or 'congruently', or attempt to capture the lack of a suitable test to capture the performance of these abilities in combination.

Author's response: The word 'simultaneously' has been included.

Reviewer's comment: Abstract Methods - the inclusion of the repeated T-agility test appears suddenly with no prior reference. If this is a key concentration of your study, I would encourage to insert earlier in the abstract along with the RIA.

Author's response: Repeated T-agility protocol now mentioned in the Background.

Reviewer's comment: Abstract Results - please specify exact $p$ values rather than just $<0.05$. CV\% can be presented to $1 \mathrm{dp}$ for brevity.

Author's response: We were unable to provide exact p-values, as several outcome measures from the repeated COD protocols, such as average time, best time, total 
time etc were presented as "performances". This same approach was used for the $r$ values as well to ensure the word limit did not exceed journal guidelines. However, we did present the CV\% to $1 \mathrm{dp}$ as requested.

Introduction

Reviewer's comment: The introduction is short and concise. However, I feel some key information that would further support the rationale for your study has been omitted. I would encourage the authors to further develop this section. A suggestion would be to expand the second paragraph into two: the first discussing the validity aspects, the second the reliability aspects. I do not expect these changes to be too onerous, but believe it would strengthen this section of your manuscript if you were to implement these changes. I have some further specific comments below.

Author's response: The second paragraph has been further developed into two as requested, with the first focusing on reliability and the second on validity.

Reviewer's comment: The opening sentence would read better if it were split into two separate sentences.

Author's response: We have simplified the first sentence by removing the context of 'agility', as we have converted all 'agility' terms to 'change-of-direction'.

Reviewer's comment: The authors should attempt to clarify during opening paragraph that they are discussing change of direction (COD) performance, rather than agility. Although COD performance is a key component of agility performance, the lack of an external stimuli prevents classification of agility. Although the tests identified by the authors in this opening paragraph have titled themselves 'agility' tests, by definition they are not. The authors should be careful in their stance here as the literature has evolved substantially since the inception of tests such as these, and appropriate classification and terminology is essential for the present study.

Young, W.; Dawson, B.; Henry, G. Agility and Change-of-Direction Speed are Independent Skills: Implications for Agility in Invasion Sports. Int. J. Sports Sci. Coach. 2015, 10, 159-169.1 Nimphius, S.; Callaghan, S.J.; Bezodis, N.E.; Lockie, R.G. Change of Direction and Agility Tests: Challenging Our Current Measures of Performance. Strength Cond. J. 2018, 40, 26-38. 2 Sheppard, J.M.; Young, W.B. Agility literature review: Classifications, training and testing. J. Sports Sci. 2006, 24, 919-932.3

Author's response: Thank you for your suggestion. As mentioned earlier, we have changed all 'agility' terms into 'change-of-direction'.

Reviewer's comment: Regarding the reliability aspect of the study, two recent papers have suggested that reliability of COD and agility may be lower in adolescent and youth athletes (as per your sample). Although these authors observed this during maximal COD/agility performance, opposed to repeated as in the present study, I believe this would be worth including and would further warrant the exploration of your research question and study. Taylor, J.M.; Cunningham, L.; Hood, P.; Thorne, B.; Irvin, G.; Weston, M. The reliability of a modified 505 test and change-of-direction deficit time in elite youth football players. Sci. Med. Footb. 2019, 3, 157-162.4

Dugdale JH, Sanders D, Hunter AM. Reliability of Change of Direction and Agility Assessments in Youth Soccer Players. Sports (Basel). 2020 Apr 18; 8 (4): 51. doi: 


\section{$10.3390 /$ sports 8040051.5}

James H. Dugdale, Calum A. Arthur, Dajo Sanders \& Angus M. Hunter (2019) Reliability and validity of field-based fitness tests in youth soccer players, European Journal of Sport Science, 19:6, 745-756, DOI: 10.1080/17461391.2018.15567396

Author's response: Thank you for your suggestion, we have included the references above in the paragraph explaining studies on the reliability of COD protocols.

Methods

Reviewer's comment: The methods report the appropriate details relating to the study design, protocols, participants, and analyses performed. However, further clarity is required in a few places throughout this section (specific comments below). Further, there are a number of oversights throughout this section that require careful attention. None of these are major points, and should take little time to amend. Yet, need to be addressed prior to potential resubmissions of this manuscript.

Author's response: Thank you for your comments, we have addressed these below.

Reviewer's comment: Figure 1: I am unsure how much this figure aids the interpretation of the study design. I personally do not feel the visual nature of this schematic clarifies the description of the trials from the text, as the study is relatively simplistic. I do not insist that the authors remove this figure, however, please consider my suggestion that it may not add value to the manuscript and may just be a "figure for the sake of a figure". Secondly, between the trials, the authors have stated that washout periods of $>7$ days were implemented. The authors should consider including the range of days (e.g. 7-14 days), as this would provide further information to the reader and allow for more accurate interpretation of the results.

Author's response: Thank you for your comment. We have decided to keep the figure to ensure clarity as we used a cross-over randomized design, which is unusual for a study examining the reliability and validity of protocols. We have included 7-14 days as a washout period as suggested in the figure.

Reviewer's comment: Can a citation or elaboration be provided to rationalise the $>15 \mathrm{~min}$ rest interval between attempts?

Author's response: A citation has been included with a study that used a 15-minute recovery period in-between repeated sprint and COD protocols in the one testing session.

Reviewer's comment: The term 'respectively' needs to be included after the explanation of the 1-10 muscle rating scale. Also, a brief sentence as to why this was collected should accompany this methodological point.

Author's response: Included as requested.

Reviewer's comment: The authors state that the warm up was the same prior to each session. I would encourage using the term 'standardised' when describing this in their methods to add certainty around their controls. Also, a citation needs to accompany the statement that the CMJ test was implemented to assess recovery. If this is what was intended by the insertion of citation 12 , I would encourage moving this citation to the end of the sentence. 
Author's response: The term 'standardised' has been included when describing the warm-up as requested in the Methods section. The citation explaining the use of CMJ to determine recovery dynamics was moved to the end of the sentence as requested.

Reviewer's comment: Participant details - participant height needs to be reported to $2 \mathrm{dp}$. Can the authors further elaborate on what the "School of Athletic Excellence" is? For example, what standard is this, is it affiliated to a specific school, or region performance programme? This may not be clear to readers.

Author's response: The participant height is reported to $2 \mathrm{dp}(1.77 \mathrm{~m})$. Further information on the excellence program has been included.

Reviewer's comment: Multistage shuttle test - a brief sentence stating how performance or a score was derived is necessary (i.e. maximum number of shuttles completed, level, etc.). This information is currently not evident.

Author's response: The VO2max was estimated based on the level completed, which has now been included in the Methods section.

Reviewer's comment: CMJ - please state the unit of measurement for this test, and to what degree (e.g. recorded in $\mathrm{cm}$ to the nearest $0.1 \mathrm{~cm}$ ).

Author's response: The CMJ was recorded to the full $\mathrm{cm}$, not to the nearest $0.1 \mathrm{~cm}$. This has now been clarified.

Reviewer's comment: 30-m sprint and agility protocols - please separate each test into a separate sentence. At present, they appear too cramped together and it is difficult to read (particularly with reference to figures and citations within). Also, please provide citations for the original studies for the T-drill and Illinois agility tests. These need to be credited within the manuscript.

Author's response: The sentences for the description of the protocols have been separated as requested. The original studies have also been included as requested.

Reviewer's comment: Repeated sprint and agility protocols - were these tests performed one after the other (what I interpret from the term 'cycles') or were they performed independently? Clarity is required to ensure the reader understands which of these interpretations is correct.

Author's response: We have reworded the term 'cycles' to 'repetitions' to improve clarity. As mentioned in Research Design and in Figure 1, RSA was conducted in Session 2, whilst RTT and RIA were conducted in the same session with 15-min rest inbetween each protocol. The Figure reinforces this design, and rewording 'cycles' to 'repetitions' improves the clarity of the protocols.

Reviewer's comment: The sentence starting "Immediately after each repeated agility cycle..." does not make sense. Please rephrase this.

Author's response: This has been reworded to, "The participant's heart rate (HR, Polar Heart Rate Monitor, Polar H10, Finland) and rating of perceived-exertion (RPE, Borg category scale 1-10) were collected at the completion of each repetition of the RSA, RTT and RIA protocols. The maximum and average HR and RPE values were then reported from the 6 repetitions" 
Reviewer's comment: Statistical analysis - Reference 16 seems a weak reference for this point. I encourage the authors to consider a more suitable citation for selecting this acceptable correlation cut off point.

Author's response: Additional reference included as requested.

Results

Reviewer's comment: The section of the results dedicated to the muscle soreness and CMJ differences (or lack thereof) should be condensed to a singular sentence and placed at the foot of the results section. This is a methodological control and not a key finding from your study, therefore, it should not feature so early and heavily within your results section. Stating that there were no differences, providing ES, and significance values would suffice. Author's response: Simplified and included at the end of the Results section as requested.

Reviewer's comment: Table 3 - I am interested in your rationale for not providing ES to demonstrate differences (or lack thereof) for your test-retest data? This would add value in my opinion. I would also encourage the authors to present CV\% data to $1 \mathrm{dp}$ both within this table and throughout the manuscript.

Author's response: Thank you for your comment. The ES was not reported as we based the measurement error on CV, with larger CV\% values exhibiting greater measurement error and poorer test-retest reliability. In our opinion, inclusion of ES would not provide any further clarity about the reliability of the tests, beyond that of CV.

Reviewer's comment: I notice that the authors use terminology such as "excellent" or "moderate" test-retest reliability, yet they have not included these threshold within the statistical analysis section of the methods. Please include this detail so that the reader understands your criteria.

Author's response: Included as requested.

Reviewer's comment: I also note that the authors say "most RIA performance measures exhibited excellent test-retest reliability...". Could the authors rephrase this to say "all RIA performance measures except... measures exhibited excellent test-retest reliability"? This would be more accurate and less ambiguous for the reader. This comment also applies later in this section where the authors refer to "a few variables...".

Author's response: Thank you for your suggestion. We have reworded this to, "Most RIA measures exhibited excellent test-retest reliability (ICC $=0.92-0.97$ ), good levels of agreement (ratio LOA $=1.05-1.06$ ) and low measurement error $(C V=2.17-2.68 \%$ ) (Table 3). However, FI and average RPE demonstrated moderate test-retest reliability (ICC $=0.87$ and 0.76 , respectively), poorer levels of agreement (ratio LOA $=2.57$ and 2.23, respectively) and higher measurement error (CV $=\mathbf{2 5 . 3}$ and $\mathbf{1 5 . 8 \%}$, respectively, Table 3)." The next paragraph has been rewritten to, "For the RTT, excellent test-retest reliability (ICC $=0.91$ ), good levels of agreement (ratio LOA $=1.08$ ) and low measurement error ( $C V=\mathbf{2 . 1 7 - 2 . 6 8 \% )}$ were identified for TT, BT and AT (Table 3), although maximum RPE demonstrated higher levels of measurement error (CV = 12.3\%) between Sessions 4 and 5 (Table 3)." 
Discussion

Reviewer's comment: I found the discussion well-written and thought the authors accurately interpreted and attempted to explain their findings. However, similar to the introduction, I felt this section lacked depth when discussing the main findings of the study. I encourage the authors to discuss their findings in greater depth, utilising some of the reading attached to this review.

Author's response: Thank you for your comments. The Discussion has been adjusted to address the comments below.

Reviewer's comment: I encourage the authors to utilise the first paragraph of the discussion to summarise all of their main findings, and then progress to discussing each point in turn. Author's response: Summary of findings included as requested in the first paragraph.

Reviewer's comment: The third paragraph begins "another novelty of this study", yet this appears to be the first time novelty is addressed in the discussion?

Author's response: This specific text has been deleted to improve clarity.

Reviewer's comment: The fourth paragraph can draw upon a wealth of correlational studies to further support the findings observed here.

Young WB, Dawson B, Henry GJ. Agility and Change-of-Direction Speed are Independent Skills: Implications for Training for Agility in Invasion Sports. International Journal of Sports Science \& Coaching. 2015;10(1):159-169. doi:10.1260/1747-9541.10.1.1591

Spiteri T, Nimphius S, Hart NH, Specos C, Sheppard JM, Newton RU. Contribution of strength characteristics to change of direction and agility performance in female basketball athletes. J Strength Cond Res. 2014 Sep;28(9):2415-23. doi: 10.1519/JSC.0000000000000547. PMID: 24875426.7

Gabbett, T. J., Kelly, J. N., \& Sheppard, J. M. (2008). Speed, Change of Direction Speed, and Reactive Agility of Rugby League Players. The Journal of Strength \& Conditioning Research, 22, 174-181. http://dx.doi.org/10.1519/JSC.0b013e31815ef7008

Sheppard, J.M.; Young, W.B.; Doyle, T.L.A.; Sheppard, T.A.; Newton, R.U. An evaluation of a new test of reactive agility and its relationship to sprint speed and change of direction speed. J. Sci Med. Sport 2006, 9, 342-349.9

Author's response: A sentence with relevant references listed above were included as requested.

Reviewer's comment: The conclusion should be reframed around the key objectives of the study (i.e. that it is reliable and valid). The mention of aerobic and anaerobic energy systems seems misplaced here.

Author's response: A sentence on the validity of the RIA protocols has been included.

References

Reviewer's comment: While a variety of key texts are identified and listed within the bibliography and cited throughout the manuscript, a number of key texts are omitted. I encourage the authors to further familiarise themselves with relevant studies assessing reliability and validity of COD and agility in team sport athletes, as well as the texts identified earlier within this review. 
Dos'Santos, T.; Thomas, C.; Jones, P.A.; Comfort, P. Mechanical Determinants of Faster Change of Direction Speed Performance in Male Athletes. J. Strength Cond. Res. 2017, 31, 696-705.10

Gabbett, T.J.; Kelly, J.N.; Sheppard, J.M. Speed, change of direction speed, and reactive agility of rugby league players. J. Strength Cond. Res. 2008, 22, 174-181.8

Oliver, J.L.; Meyers, R.W. Reliability and generality of measures of acceleration, planned agility, and reactive agility. Int. J. Sports Physiol. Perform. 2009, 4, 345-354.11

Author's response: Thank you for kindly providing the references. A number of these have been included in the paper as requested.

Competing Interests: No competing interests were disclosed.

The benefits of publishing with F1000Research:

- Your article is published within days, with no editorial bias

- You can publish traditional articles, null/negative results, case reports, data notes and more

- The peer review process is transparent and collaborative

- Your article is indexed in PubMed after passing peer review

- Dedicated customer support at every stage

For pre-submission enquiries, contact research@f1000.com 